Artigo Original

\title{
Correlações entre parâmetros aeróbios e desempenho em esforços intermitentes de alta intensidade
}

\author{
Carlos Augusto Kalva-Filho ${ }^{1}$ \\ João Paulo Loures ${ }^{2}$ \\ Vanessa Holtz Franco ${ }^{3}$ \\ Edson Itaru Kaminagakura ${ }^{3}$ \\ Alessandro Moura Zagatto 4 \\ Marcelo Papoti ${ }^{5}$ \\ ${ }^{1}$ Faculdade de Ciências e Tecnologias, UNESP - Univ Estadual Paulista, Campus de Presidente \\ Prudente, Departamento de Fisioterapia, Presidente Prudente, SP, Brasil \\ ${ }^{2}$ Instituto de Biociências, UNESP - Univ Estadual Paulista, Campus de Rio Claro, Departamento \\ de Educação Física, Rio Claro, SP, Brasil \\ ${ }^{3}$ Departamento de Educação Física, Universidade Estadual de Ponta Grossa, \\ Ponta Grossa, PR, Brasil \\ ${ }^{4}$ Faculdade de Ciências, UNESP - Univ. Estadual Paulista, Campus de Bauru, Departamento \\ de Educação Física, Bauru, SP, Brasil \\ ${ }^{5}$ Escola de Educação Física e Esportes, USP - Univ. de São Paulo, Ribeirão Preto, SP, Brasil
}

\begin{abstract}
Resumo: $O$ objetivo do presente estudo foi investigar as possíveis correlações entre parâmetros provenientes de um esforço intermitente de alta intensidade (RAST) e variáveis relacionadas ao metabolismo aeróbio (limiar anaeróbio; LAN, consumo máximo de oxigênio; $\mathrm{VO}_{2 \mathrm{MAx}}$ e intensidade correspondente ao $\mathrm{VO}_{2 \mathrm{MAX}} ; \mathrm{iVO} \mathrm{V}_{2 \mathrm{MAX}}$ ). Oito jogadores de futebol (16 \pm 1 anos) participaram do estudo. Os atletas foram submetidos a um teste progressivo para a determinação dos índices aeróbios e a seis esforços máximos de $35 \mathrm{~m}$ separados por 10 s de recuperação (RAST). As variáveis do RAST não foram correlacionadas ao $\mathrm{VO}_{2 \mathrm{MAX}}$ e ao LAN. Entretanto, as potência média absoluta e relativa ao peso corporal, apresentaram correlações significativas com a iVO $\mathrm{V}_{2 \operatorname{MAx}}(\mathrm{r}=0,79$ e $\mathrm{r}=0,85$, respectivamente). $\mathrm{O}$ índice de fadiga e a potência pico relativa também foram significativamente correlacionados com a iVO ${ }_{2 \max }(r=-0,57$ e $r=0,73$, respectivamente). Pode-se concluir que em esforços como o RAST, com breves períodos de recuperação, a única variável aeróbia correlacionada ao desempenho foi a $\mathrm{iVO}_{2 \mathrm{MAX}}$
\end{abstract}

Palavras-chave: Aptidão aeróbia. Esforços intermitentes de alta intensidade. Jogadores de futebol.

\section{Relationship between aerobic parameters and intermittent high-intensity effort performance}

Abstract: The purpose of the study was to examine the relationships between intermittent high-intensity efforts (RAST) parameters and variables related to aerobic metabolism (anaerobic threshold; LAN, maximal oxygen uptake; $\mathrm{VO}_{2 \mathrm{MAX}}$ and velocity correspondent to $\mathrm{VO}_{2 \mathrm{MAX}} \mathrm{i} \mathrm{VO}_{2 \mathrm{MAX}}$ ). Eight under-17 (U17) soccer players (16 \pm 1 years) participated in the study. The participants were submitted to a graded exercise test and six maximal sprints of $35 \mathrm{~m}$ with 10 seconds of passive recovery between each effort (RAST). The RAST parameters were not significant correlated with $\mathrm{VO}_{2 \mathrm{MAX}}$ and LAN. However absolute and relative mean power were significantly correlated with $\mathrm{iVO}_{2 \mathrm{MAX}}(\mathrm{r}=0.79$ e $\mathrm{r}=0.85$, respectively). Furthermore, the fatigue index and the relative peak power were significantly correlated with the $\mathrm{iVO}_{2 \mathrm{MAX}}(\mathrm{r}=-0,57$ e $r=0,73$, respectively). In conclusion, the only aerobic variable correlated with performance in consecutive efforts with brief recovery periods, such as RAST, is $\mathrm{iVO}_{2 \mathrm{MAX}}$.

Keywords: Aerobic fitness. Intermittent high-intensity effort. Soccer players.

\section{Introdução}

Em partidas de futebol de campo, frequentemente os jogadores são submetidos a exercícios intermitentes de alta intensidade, caracterizados por esforços máximos separados por curtos períodos de recuperação (MECKEL et al. 2009). Para suprir as demandas energéticas durante este tipo de esforço, a interação entre os metabolismos aeróbio e anaeróbio é de grande importância e necessária (GLAISTER, 2005).

Neste sentido, durante os períodos de esforço, a participação do metabolismo aeróbio é relativamente pequena (PAROLIN et al. 1999). Entretanto, durante os períodos de recuperação, o metabolismo aeróbio é o responsável por fatores importantes na manutenção do desempenho, 
como a remoção de fosfatos inorgânicos intracelulares $(\mathrm{Pi})$ e a recuperação dos estoques de fosfocreatina (PCr) (TOMLIN; WENGER, 2001, GLAISTER, 2005, GLAISTER et al. 2007). Além disso, quando os esforços máximos são repetidos com curtos intervalos de recuperação, a contribuição aeróbia aumenta durante o exercício, podendo ser determinante na manutenção do desempenho de alta intensidade (GLAISTER, 2005, GIRARD et al. 2011).

Desse modo, parece ser possível que melhores índices de desempenho em esforços intermitentes de alta intensidade são dependentes de uma boa aptidão aeróbia (AZIZ et al. 2000, BISHOP; EDGE, 2006). Entretanto, as correlações entre o desempenho em esforços intermitentes e variáveis relacionadas ao metabolismo aeróbio são contraditórias na literatura (MECKEL et al. 2009, AZIZ et al. 2000, SILVA et al. 2010). Parte desta inconsistência nos resultados pode ser explicada pela diferença entre os protocolos utilizados para avaliação do desempenho (MECKEL et al. 2009). Além disso, na maioria dos estudos, apenas o consumo máximo de oxigênio é associado ao desempenho (BISHOP; EDGE, 2006, MECKEL et al. 2009, $\underline{A Z I Z}$ et al. 2000), desconsiderando outras variáveis ligadas ao metabolismo aeróbio, como o limiar anaeróbio e a intensidade associada ao consumo máximo de oxigênio.

Adicionalmente, os modelos de exercícios intermitentes de alta intensidade, frequentemente são realizados em ciclo ergômetro e com períodos de recuperação relativamente longos (>20s) (GLAISTER et al. 2005, MORIN et al. 2011, GLAISTER et al. 2007, GLAISTER et al. 2006, BISHOP; EDGE, 2006), o que pode influenciar a extrapolação dos achados a modalidades que utilizam a corrida como principal forma de deslocamento e períodos curtos de recuperação entre os esforços (SPENCER et al. 2005), como é caso de alguns eventos no futebol.

Neste sentido, por respeitar a validade ecológica da maioria dos esportes coletivos e possibilitar avaliação do desempenho em esforços intermitentes de alta intensidade com breves períodos de recuperação, o RAST (running anaerobic sprint test) tem sido utilizado para avaliação anaeróbia (ZAGATTO et al. 2009) em várias modalidades esportivas (ALIZADEH et al. 2010, ROSEGUINI et al. 2008, BALČIŪNAS et al. 2006). Entretanto, recentemente foi demonstrado que os parâmetros provenientes do RAST não foram associados com a capacidade anaeróbia, assumida como o máximo déficit acumulado de oxigênio (MAOD) (KAMINAGAKURA et al. 2012).

Embora pareça ser um bom modelo para o estudo do desempenho em esforços intermitentes de alta intensidade, respeitando a ação motora e as características da maioria das modalidades coletivas, poucos estudos investigaram as possíveis associações de variáveis relacionadas ao metabolismo aeróbio em esforços como o RAST. Além disso, parece ser relevante para o treinamento em modalidades coletivas, que as interações entre o metabolismo aeróbio e o desempenho em esforços semelhantes aos realizados durante os treinamentos e competições sejam melhores estudadas. Com isso, o objetivo do presente estudo foi determinar as possíveis correlações entre o desempenho no RAST e índices ligados ao metabolismo aeróbio.

\section{Materiais e Método}

\section{Participantes}

Participaram voluntariamente deste estudo oito futebolistas da categoria sub-17, com idade média de $16 \pm 1$ anos, estatura de $174,5 \pm 3,7 \mathrm{~cm}$ e massa corporal de $64,8 \pm 4,7 \mathrm{~kg}$. Os responsáveis pelos atletas foram informados sobre os riscos e benefícios do presente estudo e somente foram incluídos nas análises aqueles que concordaram por escrito com o termo de consentimento livre e esclarecido. Os procedimentos deste estudo foram aprovados pelo comitê de ética em pesquisa da Instituição (protocolo no 2982) e aplicados em conformidade com a Declaração de Helsinque.

\section{Delineamento experimental}

Os atletas foram submetidos a duas sessões de avaliação, separadas por um intervalo mínimo de $24 \mathrm{~h}$. Na primeira sessão os participantes realizaram um teste incremental máximo para a determinação do consumo máximo de oxigênio $\left(\mathrm{VO}_{2 \mathrm{MAX}}\right)$, da intensidade correspondente ao $\mathrm{VO}_{2 \text { MAX }}\left(\mathrm{iVO}_{2 \mathrm{MAX}}\right.$ ) e do limiar anaeróbio (LAN), que foram consideradas como variáveis aeróbias. $\mathrm{Na}$ segunda sessão os atletas foram submetidos ao exercício intermitente de alta intensidade, composto por seis esforços máximos de $35 \mathrm{~m}$ intercalados a um período de recuperação passiva de 10s (RAST), utilizando chuteiras na grama. Durante 0 teste incremental 
(imediatamente após cada estágio de exercício e ao final do teste) e após o sexto esforço do RAST, 25 microlitros de sangue foram coletados do lóbulo da orelha para a determinação das concentrações de lactato ([Lac]) utilizando um analisador eletroquímico YSI 1500 SPORT (YSI, Yellow Spring, Ohio, USA).

\section{Determinação das variáveis aeróbias}

Para a determinação das variáveis aeróbias, após um aquecimento de $7 \mathrm{~min}$ a $8 \mathrm{Km} \cdot \mathrm{h}^{-1}$, os participantes foram submetidos a um teste incremental exaustivo em esteira motorizada (Inbramed Super Master ATL, Inbrasport, Porto Alegre, Brasil) com velocidade inicial de $10 \mathrm{Km} \cdot \mathrm{h}^{-1}$ e incrementos de $1 \mathrm{Km} \cdot \mathrm{h}^{-1}$ a cada três minutos. A inclinação da esteira foi mantida constante a $1 \%$. Durante o teste, a frequência cardíaca (FC; Polar, vantage NV, Finlândia) e as variáveis ventilatórias e respiratórias (True-One 2400, ParvoMedics, East Sandy, Utah, USA) foram monitoradas constantemente respiração à respiração.

$\mathrm{O} \mathrm{VO}_{2 \text { MAX }}$ foi assumido como a maior média do consumo de oxigênio $\left(\mathrm{VO}_{2}\right)$ dos últimos $30 \mathrm{~s}$ de exercício, quando pelo menos dois dos quatro critérios foram observados: 1) [Lac] $\geq 8 \mathrm{mM}$; 2) frequência cardíaca $\geq 90 \%$ da máxima predita (220-idade); 3) coeficiente respiratório $(\mathrm{QR}) \geq$ 1,10 e 4) variação no $\mathrm{VO}_{2}$ entre o penúltimo e ultimo estágio de exercício menor que 2,1 $\mathrm{ml} / \mathrm{kg} / \mathrm{min}$. A $\mathrm{iVO}_{2 \mathrm{MAX}}$ foi considerada como a menor intensidade em que o $\mathrm{VO}_{2 \mathrm{MAX}}$ foi atingido. O limiar anaeróbio (LAN) foi assumido como a intensidade correspondente a concentração de 3,5 mM, obtida da relação exponencial entre as [Lac] e as intensidades de cada estágio.

\section{Avaliação do desempenho em esforços intermitentes de alta intensidade}

O desempenho em esforços intermitentes de alta intensidade foi avaliado por meio do RAST. Previamente a realização dos esforços, a massa corporal total (incluindo vestimentas) foi mensurada por meio de uma balança digital (TANITA UM080, Brasil). O RAST consistiu na realização de seis corridas máximas na distância de $35 \mathrm{~m}$, separadas por um período de recuperação passiva de 10s. O registro de tempo foi realizado a cada esforço (Timex ${ }^{\circledR}$, modelo 85103) para a determinação da potência gerada a cada corrida (Potência=massa corporal $\mathrm{x}$ Distância ${ }^{2} /$ tempo $^{3}$ ) (ZAGATTO et al. 2009).
Como variáveis do RAST foram determinadas as potências pico (PP), potência média $(P M)$ e o índice de fadiga (IF) (IF (\%) $=((\mathrm{PP}-$ potência mínima) x 100) / PP). Além disso, por meio da relação entre a distância e o tempo de esforço, foram determinadas a velocidade máxima $\left(\mathrm{V}_{\mathrm{MAX}}\right)$ $\mathrm{e}$ a velocidade média $\left(\mathrm{V}_{\mathrm{MED}}\right)$. Após o sexto esforço as concentrações sanguíneas de lactato foram monitoradas por sete minutos para a determinação das concentrações de lactato pico ([Lac $]_{\text {PICO }}$.

\section{Tratamento Estatístico}

Os resultados estão apresentados em médias \pm desvio padrão e intervalo de confiança de $95 \%$ (IC95\%) para cada variável. A normalidade dos dados foi testada e confirmada pelo teste de Shapiro-Wilk, o que permitiu a análise estatística paramétrica. As possíveis correlações entre as variáveis aeróbias e os parâmetros provenientes do RAST foram evidenciadas por meio do teste de correlação de Pearson. $\mathrm{Na}$ interpretação dos valores de correlação, além da significância $(p<0,05)$, os coeficientes de correlação $(r)$ foram classificados em: muito fraco $(0,0-0,2)$, fraco $(0,2-0,4)$, moderado $(0,4-0,7)$, forte $(0,7-0,9)$ e muito forte $(0,9-1,0)$ (ROWNTREE et al. 1991). Todas as análises foram realizadas com o auxilio do pacote estatístico STATISTICA 7 (Statsoft, EUA), e em todos os casos o nível de significância foi pré-fixado em $p<0,05$.

\section{Resultados}

Todos os participantes preencheram pelo menos dois dos três critérios freqüentemente exigidos para obtenção do $\mathrm{VO}_{2 \mathrm{MAX}}(\mathrm{QR}=1,2 \pm 0,1$; $\mathrm{FC}=95,2 \pm 2,8 \%$ da máxima; $[\mathrm{Lac}]=8,9 \pm 2,1 \mathrm{mM}$ ). Os valores de LAN, $V_{2 \operatorname{MAX}}$ e $\mathrm{iVO}_{2 \mathrm{MAX}}$, são apresentados na tabela 1 .

Os valores de PP, PM, IF, V VAX e $V_{\text {MED }}$ provenientes do RAST são apresentados na tabela 2. Nenhum parâmetro do RAST foi significativamente correlacionado ao LAN e ao $\mathrm{VO}_{2 \text { MAX. Entretanto, para a iVO }}$ MAX, com exceção da PP absoluta (W) $(p=0,06)$ e da [Lac] $]_{\text {IICO }}$ $(p=0,79)$, todas as outras variáveis do RAST apresentaram significativas correlações, que variaram de moderadas (IF) a fortes (PP (W. $\left.\mathrm{kg}^{-1}\right)$, $\mathrm{PM}, \mathrm{V}_{\text {MAX }}$ e $\mathrm{V}_{\text {MED }}$ ). Todas as correlações foram apresentadas na tabela 3 . 
Tabela 1. Valores médios, desvio padrão (DP) e o intervalo de confiança (IC95\%) das variáveis ligadas ao metabolismo aeróbio, determinadas durante o teste incremental.

\begin{tabular}{|c|c|c|c|}
\hline & Média & DP & IC95\% \\
\hline $\mathrm{VO}_{2 \operatorname{MAX}}\left(\right.$ L. $\left.\min ^{-1}\right)$ & 3.3 & 0.5 & $2.9-3.8$ \\
\hline $\mathrm{VO}_{2 \operatorname{MAX}}\left(\mathrm{mL} \cdot \mathrm{kg}^{-1} \cdot \mathrm{min}^{-1}\right)$ & 51,6 & 8,7 & $44,3-58,9$ \\
\hline $\mathrm{iVO}_{2 \operatorname{MAX}}\left(\mathrm{Km}^{-\mathrm{h}^{-1}}\right)$ & 15,4 & 1,4 & $14,3-16,6$ \\
\hline LAN $\left(\mathrm{Km} \cdot \mathrm{h}^{-1}\right)$ & 11,4 & 2,5 & $9,3-13,5$ \\
\hline LAN (\%VO & 73,5 & 12,1 & $63,4-83,7$ \\
\hline
\end{tabular}

Tabela 2. Valores médios, desvio padrão (DP) e o intervalo de confiança (IC95\%) das variáveis provenientes do RAST.

\begin{tabular}{cccc}
\hline & Média & DP & IC95\% \\
\hline PP $(\mathrm{W})$ & 667,3 & 67,0 & $611,3-723,4$ \\
PP $\left(\mathrm{W} . \mathrm{kg}^{-1}\right)$ & 10,3 & 1,2 & $9,3-11,3$ \\
PM $(\mathrm{W})$ & 555,9 & 74,7 & $493,4-618,4$ \\
PM $\left(\mathrm{W} . \mathrm{kg}^{-1}\right)$ & 8,6 & 1,2 & $7,6-9,6$ \\
IF $(\%)$ & 34,1 & 6,6 & $28,5-39,6$ \\
V $_{\text {MAX }}\left(\mathrm{m} . \mathrm{s}^{-1}\right)$ & 7,1 & 0,3 & $6,9-7,4$ \\
V $_{\text {MED }}\left(\mathrm{m.s}^{-1}\right)$ & 6,7 & 0,3 & $6,4-6,9$ \\
{$[$ Lac]PICO $(\mathrm{Mm})$} & 9,9 & 3,2 & $7,2-12,5$ \\
\hline
\end{tabular}

PP: potência pico; PM: potência média; Pmin: potência mínima; IF: índice de fadiga; $\mathrm{V}_{\mathrm{MAx}}$ : velocidade máxima; $\mathrm{V}_{\mathrm{MED}}$ : velocidade média; $\mathrm{V}_{\mathrm{MIN}}$ : velocidade mínima; [Lac] $]_{\mathrm{PICO}}$ : concentrações de lactato pico.

Tabela 3. Coeficientes de correlação ( $r$ ) entre os parâmetros provenientes do RAST e o consumo máximo de oxigênio $\left(\mathrm{VO}_{2 \mathrm{MAX}}\right)$, limiar anaeróbio ( $\mathrm{LAN}$ ) e intensidade correspondente ao $\mathrm{VO}_{2 \mathrm{MAX}}\left(\mathrm{iVO}_{2 \mathrm{MAX}}\right)$.

\begin{tabular}{|c|c|c|c|c|c|}
\hline & $V_{\text {MAX }}$ & & LAN & & $\mathrm{iVO}_{2 \mathrm{MAX}}$ \\
\hline & $\left(\right.$ L.min $\left.\min ^{-1}\right)$ & $\left(\mathrm{mL} \cdot \mathrm{kg}^{-1} \cdot \mathrm{min}^{-1}\right)$ & $\left(\mathrm{Km} \cdot \mathrm{h}^{-1}\right)$ & $\left(\% \mathrm{VO}_{2 \text { MAX }}\right)$ & $\left(\mathrm{Km} \cdot \mathrm{h}^{-1}\right)$ \\
\hline PP & 0,45 & 0,34 & 0,58 & 0,44 & 0,68 \\
\hline PP relativa & 0,45 & 0,58 & 0,57 & 0,40 & $0,73^{*}$ \\
\hline PM & 0,63 & 0,50 & 0,46 & 0,21 & $0,79^{\star}$ \\
\hline PM relativa & 0,61 & 0,67 & 0,47 & 0,20 & $0,85^{\star}$ \\
\hline IF & $-0,57$ & $-0,37$ & $-0,21$ & 0,02 & $-0,57^{*}$ \\
\hline$V_{\text {MAX }}$ & 0,46 & 0,59 & 0,57 & 0,40 & $0,74^{*}$ \\
\hline$V_{M E D}$ & 0,62 & 0,69 & 0,48 & 0,22 & $0,86^{*}$ \\
\hline$[\mathrm{LaC}]_{\mathrm{PICO}}$ & 0,18 & 0,34 & 0,05 & 0,02 & 0,11 \\
\hline
\end{tabular}

\section{Discussão}

O principal achado do presente estudo foi as significativas correlações encontradas entre a $\mathrm{iVO}_{2 \text { MAX }}$ e o desempenho no RAST.

Significativas correlações entre o $\mathrm{VO}_{2 \mathrm{MAX}}$ e $\mathrm{O}$ desempenho têm sido encontradas em estudos que utilizaram cinco (BISHOP et al. 2004, BISHOP; EDGE, 2006), sete (SILVA et al. 2010) e oito (AZIZ et al. 2000) esforços máximos, porém separados por um período de recuperação relativamente superior (20-30s) ao aplicado no presente estudo. Além disso, quando o período de recuperação é diminuído (<20s), o $V \mathrm{VO}_{2 \mathrm{MAx}}$ é correlacionado ao desempenho em modelos intermitentes com um número superior de repetições (entre 12 a 20 repetições) (MECKEL et al. 2009, GLAISTER et al. 2006). Neste sentido, estas correlações parecem ser dependentes de um grande número de repetições e/ou de um período de recuperação relativamente longo, o que não ocorre em esforços semelhantes ao RAST.

Diferentemente do $\mathrm{VO}_{2 \mathrm{MAX}}$ que é determinado por fatores centrais (SILVA et al. 2010), o LAN é relacionado a fatores periféricos, como o transporte de ions $\mathrm{H}^{+}$e a ressintese de $\mathrm{PCr}$ (BILLAT et al. 2003), que também são considerados fatores essenciais para 0 desempenho em esforços intermitentes de alta intensidade (GLAISTER, 2005). Entretanto, poucos estudos investigam as correlações do LAN e este tipo de exercício.

Silva et al. (2010), evidenciaram significativas correlações entre o LAN e o desempenho em sete 
esforços máximos de $34,2 \mathrm{~m}$ realizados a cada 25s. Entretanto, Bishop et al. (2004), observaram significativas correlações entre 0 LAN e o desempenho (5x6s, com $30 \mathrm{~s}$ de intervalo), apenas quando esta variável foi expressa em relação ao consumo de oxigênio $\left(\mathrm{mL} \cdot \mathrm{kg}^{-1} \cdot \mathrm{min}^{-1}\right)$, não ocorrendo 0 mesmo em função da intensidade (W). Além disso, os estudos supracitados (SILVA et al. 2010; BISHOP et al. 2004), embora tenham utilizado esforços com duração semelhante $(\sim 5 \mathrm{~s})$, realizaram períodos de recuperação superiores (25 e 30s) aos aplicados durante o RAST. Com isso, parecem ser necessários períodos de recuperação mais prolongados para que a capacidade aeróbia contribua significantemente no desempenho neste tipo de exercício.

Em conjunto, estes achados parecem evidenciar que as correlações do LAN e do $\mathrm{VO}_{2 \mathrm{MAX}}$ ao desempenho em esforços intermitentes de alta intensidade parecem ser influenciadas por fatores como o número de repetições, a forma de expressão das variáveis e, principalmente, a duração dos períodos de recuperação. Com isso, as correlações não significativas evidenciadas no presente estudo, podem ser explicadas pelo número reduzido de esforços (seis) e pela duração insuficiente dos períodos de recuperação (10s), aplicados durante o RAST.

Assim como ocorre com o LAN, poucos estudos investigaram as relações da $\mathrm{iVO}_{2 \mathrm{MAX}} \mathrm{com}$ o desempenho em esforços intermitentes de alta intensidade. Corroborando com os achados da presente investigação, Silva et al. (2010) observaram significativas, porém fracas, correlações da $\mathrm{iVO}_{2 \mathrm{MAX}}$ com o tempo médio de esforço $(r=-0,38)$ e o índice de decaimento da velocidade $(r=-0,49)$, observados após sete esforços de $\sim 34 \mathrm{~m}$ realizados a cada $25 \mathrm{~s}$. Recentemente, Thébault et al. (2011) observaram diferentes coeficientes de corelação entre a $\mathrm{iVO}_{2 \text { MAX }}$ e o desempenho, sendo observado valores superiores quando a variável aeróbia foi determinada por meio de um teste progressivo com mudanças de direção e momentos de aceleração e desaceleração. Com isso, embora possam sofrer influência do método utilizado para a determinação da $\mathrm{iVO}_{2 \mathrm{MAX}}$ (THÉBAULT et al. 2011), as correlações entre este índice aeróbio e o desempenho parecem não sofrer influência do modelo de esforço intermitente utilizado.
Principalmente pelos diferentes custos energéticos envolvidos (SASSI et al. 2011), uma possível limitação do presente estudo pode ter sido a utilização de diferentes superfícies para a determinação das variáveis aeróbias (esteira) e dos parâmetros provenientes do RAST (grama). Entretanto, o RAST foi realizado na grama e utilizando chuteiras para que as condições de avaliação fossem condizentes com a rotina de treinamento e competição imposta para jogadores de futebol. Além disso, a utilização da esteira permitiu a determinação do consumo de oxigênio de maneira direta (ParvoMedics, East Sandy, Utah, USA), implicando em valores mais robustos para esta variável. Neste sentido, futuras investigações devem preocupar-se com a padronização da superfície utilizada para a determinação das variáveis aeróbias e do desempenho em esforços repetitivos.

Em resumo, os achados do presente estudo demonstraram que embora significativas, as correlações entre variáveis relacionadas ao metabolismo aeróbio e o desempenho em esforços intermitentes de alta intensidade parecem ser fortemente influenciadas pelo modelo de exercício intermitente utilizado, sendo que para esforços com um número reduzido de repetições e curtos períodos de recuperação a $i \mathrm{VO}_{2 \mathrm{MAX}}$ foi a única variável relacionada ao desempenho.

\section{Referências}

ALIZADEH, R.; HOVANLOO, F.; SAFANIA, A.M. The relationship between aerobic power and repeated sprint ability in young soccer players with different levels of vo2 max. Journal of Physical Education and Sport, Pitesti, v. 27, n. 2, p. 8692, 2010.

AZIZ, A.R.; CHIA, M.; TEH, K.C. The relationship between maximal oxygen uptake and repeated sprint performance indices in field hockey and soccer players. The Journal of Sports Medicine Physical and Fitness, Torino, v. 40, n. 3, p. 195200, 2000.

BALČIŪNAS, M.; STONKUS, S.; ABRANTES, C.; SAMPAIO, J. Long term effects of different training modalities on power, speed, skill and anaerobic capacity in young male basketball players. Journal of Sports Science and Medicine, Bursa, n. 5, p. 163-170, 2006.

BILLAT, V.L.; SIRVENT, P.; PY,G.; KORALSZTEIN, J.P.; ANDMERCIER, J. 
The concept of maximal lactate steady state: a bridge between biochemistry, physiology and sport science. Sports Medicine, Auckland, v. 33, n. 6, p. 406-426, 2003.

BISHOP, D.; EDGE, J.; GOODMAN, C. Muscle buffer capacity and aerobic fitness are associated with repeated-sprint ability in women. European Journal of Applied Physiology, Basel, v. 92, n. 5, p.540-7, 2004.

BISHOP, D.; EDGE, J. Determinants of repeatedsprint ability in females matched for single-sprint performance. European Journal of Applied Physiology, Basel, v. 97, n. 4, p. 373-379, 2006.

KAMINAGAKURA, E.I.; REDKVA, P.E.; GOMES, E.B.; LOURES, J.P.; KALVA-FILHO, C.A.; ZAGATTO, A.M.; PAPOTI, M. The anaerobic sprint running test can be used as a predictor of anaerobic capacity? Journal of Exercise Physiology, Duluth, v. 15, n. 2, p. 9099, 2012. Disponível em:

$<$ http://faculty.css.edu/tboone2/asep/JEPonlineApr il2012Kaminagakura Zagatto.pdf> Acesso em: 22 mai. 2012.

GIRARD, O.; MENDEZ-VILLANUEVA, A.; BISHOP, D. Repeated-sprint ability - part I: factors contributing to fatigue. Sports Medicine. Auckland, v. 41, n. 8, p. 673-694, 2011.

GLAISTER, M. Multiple Sprint Work: Physiological Responses, Mechanisms of Fatigue and the Influence of Aerobic Fitness. Sports Medicine, Auckland, v. 35, n. 9, p. 757-777, 2005.

GLAISTER, M.; STONE, M.H.; STEWART, A.M.; HUGHES, M.G.; MOIR, G.L. Aerobic and anaerobic correlates of multiple sprint cycling performance. The Journal of Strength and Conditioning Research, Philadelphia, v. 20, n. 4, p. 792-798, 2006.

GLAISTER, M.; STONE, M.H.; STEWART, A.M.; HUGHES, M.G.; MOIR, G.L. The influence of endurance training on multiple sprint cycling performance. The Journal of Strength and Conditioning Research, Philadelphia, v. 21, n. 2, p. 606-612, 2007.

MECKEL, Y.; MACHNAI, O.; ELIAKIM, A. Relationship among repeated sprint tests, aerobic fitness, and anaerobic fitness in elite adolescent soccer players. The Journal of Strength and Conditioning Research. Philadelphia, v. 23, n. 1, p. 163-169, 2009.

MORIN, J.B.; DUPUY, J.; SAMOZINO, P. Performance and fatigue during repeated sprints: what is the appropriate sprint dose? The Journal of Strength and Conditioning Research, Philadelphia, v. 25, n. 7, p. 1918-1924, 2011.

PAROLIN, M.L.; CHESLEY, A.; MATSOS, M.P.; SPRIET, L.L.; JONES, N.L.; HEIGENHAUSER, G.J.F. Regulation of skeletal muscle glycogen phosphorylase and PDH during maximal intermittent exercise. American Journal of Physiology, Bethesda, v. 277, n. 5, p. 890-900, 1999.

ROSEGUINI, A.Z.; SILVA, A.S.; GOBATTO, C.A.; Determinações e relações dos parâmetros anaeróbios do RAST, do limiar anaeróbio e da resposta lactacidêmica obtida no inicio, no intervalo e ao final de uma partida oficial de handebol. Revista Brasileira de Medicina do Esporte, São Paulo, v. 14, n. 1, p. 46-50, 2008. Disponível em: $<$ http://www.scielo.br/pdf/rbme/v14n1/a09v14n1.p df> Acesso em: 22 mai. 2012.

\section{ROWNTREE,D. Statistics without tears.} London: Penguin, 1991.

SASSI, A.; STEFANESCU, A.; MENASPA, P.; BOSIO, A.; RIGGIO, M.; RAMPININI, E. The cost of running on natural grass and artificial turf surfaces. The Journal of Strength and Conditioning Research, Philadelphia, v. 25, n. 3, p. 606-611, 2011.

SILVA, J.F.; GUGLIELMO, L.G.; BISHOP, D. Relationship between different measures of aerobic fitness and repeated-sprint ability in elite soccer players. The Journal of Strength and Conditioning Research, Philadelphia, v. 24, n. 8, p. 2115-2121, 2010.

SPENCER, M.; BISHOP, D.; DAWSON, B.; GOODMAN, C. Physiological and metabolic responses of repeated-sprint activities: specific to field-based team sports. Sports Medicine.

Auckland, v. 35, n. 12, p. 1025-44, 2005.

THÉBAULT, N.; LÉGER, L.A.; PASSELERGUE, $P$. Repeated-sprint ability and aerobic fitness. The Journal of Strength and Conditioning Research, Philadelphia, v. 25, n. 10, p. $2857-$ 2865, 2011.

TOMLIN, D.L.; WENGER, H.A. The Relationship Between Aerobic Fitness and Recovery from High Intensity Intermittent. Sports Medicine, v. 31, n. 1, p. 1-11, 2001.

ZAGATTO, A.M.; BECK, W.R.; GOBATTO, C.A. Validity of the Running Anaerobic Sprint Test for Assessing Anaerobic Power and Predicting ShortDistance Performances. The Journal of 
Strength and Conditioning Research,

Philadelphia, v. 23, n. 6, p. 1820-1827, 2009.

\section{Endereço:}

Carlos Augusto Kalva Filho

Rua Pastor Fugmann, 247 Bairro: Nova Rússia

Ponta Grossa PR Brasil

84070-030

Telefone: (42) 9941-0362

e-mail: kalvafilho@yahoo.com.br

Recebido em: 23 de maio de 2012.

Aceito em: 07 de abril de 2013.

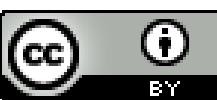

Motriz. Revista de Educação Física. UNESP, Rio Claro,

SP, Brasil - elSSN: 1980-6574 - está licenciada sob Creative Commons - Atribuição 3.0 